\title{
Electron-Positron Interaction in Jellium: Optimization of the Perturbed Hypernetted-Chain Approach
}

\author{
H. STACHOWIAK AND E. BorońsKI
}

W. Trzebiatowski Institute for Low Temperature and Structure Research Polish Academy of Sciences, P.O.Box 1410, 50-950 Wrocław 2, Poland

The perturbed hypernetted-chain approach to electron-positron interaction in jellium consists in assuming the wave function of the system in the form of a Slater determinant built of single-electron functions presented as $\psi_{\boldsymbol{k}}(\boldsymbol{s})=w(s) \varphi_{\boldsymbol{k}}(\boldsymbol{s}) . s$ is the distance between the electron and the positron. The function $w(s)$ is obtained by solving the equation of Gondzik and Stachowiak. The function $\varphi_{\boldsymbol{k}}(\boldsymbol{s})$ is computed by applying a self-consistent Born approximation. The idea of the paper is to modify the function $w(s)$ in such a way as to obtain from the Born approximation as precise results as possible. It is shown that the influence of such modifications on physical predictions is small. This shows how good is the perturbed hypernetted-chain approach. The annihilation rates obtained in such a way decrease somewhat, becoming closer to experimental expectations.

PACS numbers: 71.60.+z, 78.70.Bj, 71.10.Ca

\section{Introduction}

As remarked already in Refs. [1, 2], the best calculations of positron annihilation rates in jellium lead to the results higher by approximately 8 to $16 \%$ than to the ones expected on the basis of numerous measurements performed in real metals ( $8 \%$ concerns perturbed hypernetted-chain (PHNC) approach).

The idea of the present paper is to modify the PHNC approach developed in Refs. [3-5] in the hope that a better theory would lead to lower annihilation rates. At the same time such calculations would help to find how good is the self-consistent Born approximation used in the PHNC approach.

Additional information concerning the theory of electron-positron interaction in jellium can be found in Ref. [6].

\section{The two-particle approximation}

It is usually assumed that the interaction of electrons in an electron gas with the positron consists in the interaction of particular electronic states with the positron, and momentum conservation occurs in this two-particle system. This is 
the reason of the usefulness of positron annihilation in studies of the electronic structure.

The wave function of the jellium-one positron system is assumed as a Slater determinant built of functions $\psi_{\boldsymbol{k} \sigma}\left(\boldsymbol{r}_{\mathrm{e}}, \boldsymbol{r}_{\mathrm{p}}\right)$. Here $\boldsymbol{r}_{\mathrm{e}}$ and $\boldsymbol{r}_{\mathrm{p}}$ are electron and positron coordinates, respectively. The electron spin index $\sigma$ will be omitted unless necessary. The functions $\psi_{\boldsymbol{k}}$ describe the scattering of the electron plane wave on the screened positron and obey the following equation (all the quantities throughout the paper are given in Hartree atomic units):

$$
\left(-\frac{1}{2} \nabla_{\mathrm{e}}^{2}-\frac{1}{2} \nabla_{\mathrm{p}}^{2}+V\left(\left|\boldsymbol{r}_{\mathrm{e}}-\boldsymbol{r}_{\mathrm{p}}\right|\right)\right) \psi_{\boldsymbol{k}}=\frac{k^{2}}{2} \psi_{\boldsymbol{k}}
$$

The screened Coulomb potential $V(s)$ will be computed self-consistently.

In reduced coordinates $\psi_{\boldsymbol{k}}$ can be written as

$$
\psi_{\boldsymbol{k}}\left(\boldsymbol{r}_{\mathrm{e}}, \boldsymbol{r}_{\mathrm{p}}\right)=\frac{1}{\sqrt{\Omega}} \exp (\mathrm{i} \boldsymbol{k} \boldsymbol{R}) u_{\boldsymbol{k}}(\boldsymbol{s})
$$

where

$$
\boldsymbol{R}=\frac{1}{2}\left(\boldsymbol{r}_{\mathrm{e}}+\boldsymbol{r}_{\mathrm{p}}\right), \quad s=\boldsymbol{r}_{\mathrm{e}}-\boldsymbol{r}_{\mathrm{p}}
$$

and $u_{\boldsymbol{k}}$ satisfies the equation

$$
\left(-\frac{1}{2} \nabla^{2}+\frac{1}{2} V(s)\right) u_{k}=\frac{k^{2}}{8} u_{k}
$$

$\Omega$ is the volume of the sample.

Following the usual way of dealing with such problems [7], we obtain $u_{\boldsymbol{k}}$ in the form as:

$$
u_{\boldsymbol{k}}(\boldsymbol{s})=\frac{1}{\sqrt{\Omega}}\left[\exp \left(\mathrm{i} \frac{\boldsymbol{k}}{2} \boldsymbol{s}\right)+\sum_{l=0}^{l_{M}}(2 l+1) i^{l} \beta_{l}\left(\frac{k}{2}, s\right) P_{l}(\cos \vartheta)\right],
$$

where $\vartheta$ is the angle between $\boldsymbol{s}$ and $\boldsymbol{k}$,

$$
\beta_{l}\left(\frac{k}{2}, s\right)=\exp \left(\mathrm{i} \delta_{l} \frac{k}{2}\right) b_{l}\left(\frac{k}{2}, s\right)-j_{l}\left(\frac{k}{2} s\right),
$$

$\delta_{l}$ indicates phase shifts, $j_{l}$ the appropriate Bessel function and $b_{l}$ satisfies the relation

$$
R_{l}\left(\frac{k}{2}, s\right)=(2 l+1) i^{l} \exp \left(\mathrm{i} \delta_{l}\left(\frac{k}{2}\right)\right) b_{l}\left(\frac{k}{2}, s\right),
$$

where $R_{l}$ is the solution of the radial part of Eq. (4).

It seems that $l_{M}=3$ is sufficient to describe the screening of a positron.

Introducing (5) into (2) we get

$$
\begin{aligned}
\psi_{\boldsymbol{k}}\left(\boldsymbol{r}_{\mathrm{e}}, \boldsymbol{r}_{\mathrm{p}}\right)=\frac{\exp \left(\mathrm{i} \boldsymbol{k} \boldsymbol{r}_{\mathrm{p}}\right)}{\Omega} \\
\quad \times\left[\exp (\mathrm{i} \boldsymbol{k} \boldsymbol{s})+\exp \left(\mathrm{i} \frac{\boldsymbol{k}}{2} \boldsymbol{s}\right) \sum_{l=0}^{l_{M}}(2 l+1) i^{l} \beta_{l}\left(\frac{k}{2}, s\right) P_{l}(\cos \vartheta)\right] .
\end{aligned}
$$

Since the total momentum of the system vanishes, the Slater determinant of the system can be built of functions as follows:

$$
\psi_{\boldsymbol{k}}(\boldsymbol{s})=\frac{1}{\sqrt{\Omega}}\left[\exp (\mathrm{i} \boldsymbol{k s})+\exp \left(\mathrm{i} \frac{\boldsymbol{k}}{2} \boldsymbol{s}\right) \sum_{l=0}^{l_{M}}(2 l+1) i^{l} \beta_{l}\left(\frac{k}{2}, s\right) P_{l}(\cos \vartheta)\right] .
$$




\section{The perturbed hypernetted-chain approach}

The functions $\psi_{\boldsymbol{k}}(\boldsymbol{s})$ can be expressed in the following form:

$$
\psi_{\boldsymbol{k}}(\boldsymbol{s})=\frac{w(s)}{\sqrt{\Omega}}\left(\exp (\mathrm{i} \boldsymbol{k} \boldsymbol{s})+v_{\boldsymbol{k}}(\boldsymbol{s})\right),
$$

where

$$
v_{\boldsymbol{k}}(\boldsymbol{s})=\frac{1}{\Omega} \sum_{\boldsymbol{q}} A_{\boldsymbol{k}}(\boldsymbol{q}) \exp (\mathrm{i}(\boldsymbol{k}+\boldsymbol{q}) \boldsymbol{s}) .
$$

This allows the scattering of electrons to occupied momentum states lying inside the Fermi sphere, violating in this way the Pauli exclusion principle. Of course, there is no reason to worry about that as long as the wave function of the system remains antisymmetric. However, this complicates the computation of the electronic charge screening the positron and needs additional considerations unlike in the case of screening a heavy particle. Several approaches to this problem have been proposed in Refs. [8-12].

Kallio et al. [10] adopting after Zabolitzky [13] the Lado approximation, proposed a particularly simple way of minimizing the trial function of Lantto [10]. This resulted in the equation

$$
\left(-\nabla^{2}+V(\boldsymbol{s})\right) w(\boldsymbol{s})=0
$$

for the electron density amplitude $w(\boldsymbol{s})$. $V(s)$ remained, however, a rather complicated functional of the electron distribution.

Gondzik and Stachowiak proposed to simply replace this potential by its Kohn-Sham form [12]

$$
V_{0}(\boldsymbol{s})=-\frac{1}{s}+\rho_{0} \int \mathrm{d} \boldsymbol{s}^{\prime} \frac{w^{2}\left(s^{\prime}\right)-1}{\left|\boldsymbol{s}-\boldsymbol{s}^{\prime}\right|}+V_{\mathrm{xc}}\left\{w^{2}(s) \rho_{0}\right\}-V_{\mathrm{xc}}\left\{\rho_{0}\right\},
$$

where $V_{\mathrm{xc}}\{\rho\}$ is the exchange-correlation potential (e.g. according to Hedin and Lundqvist [14]) corresponding to the electron density $\rho . \rho_{0}$ is the electron density.

The computation of the positron annihilation rate in the above formalism is particularly simple and consists in solving a single nonlinear integro-differential equation. The results are reasonable and easy to obtain in the whole range of electron densities.

The approaches of Lantto [9], of Kallio et al. [11, 10], and of Gondzik and Stachowiak [12] neglect completely the dependence of the electron-positron interaction on the momentum state of the electron. This suggests that assuming that every electronic state is scattered on the positron in the same way, it is a quite good approximation.

Basing on this observation, the wave function $\psi_{\boldsymbol{k}}(\boldsymbol{s})$ was assumed in Ref. [3] in the form as:

$$
\psi_{\boldsymbol{k}}(\boldsymbol{s})=w(s) \varphi_{\boldsymbol{k}}(\boldsymbol{s})
$$

The function $w(s)$ was chosen as the solution of Eq. (12) with the potential (13). $\varphi_{\boldsymbol{k}}(\boldsymbol{s})$ was assumed in the form as: 


$$
\varphi_{\boldsymbol{k}}(s)=\frac{1}{\sqrt{\Omega}}\left[\exp (\mathrm{i} \boldsymbol{k s})+v_{\boldsymbol{k}}(\boldsymbol{s})\right]
$$

while assuming the smallness of $v_{\boldsymbol{k}}(\boldsymbol{s})$. The wave function of the system takes the following form:

$$
\Psi\left(s_{1}, \ldots, s_{N}\right)=\prod_{i=0}^{N} w\left(s_{i}\right)\left|\begin{array}{cccc}
\varphi_{1}\left(s_{1}\right) & \varphi_{1}\left(s_{2}\right) & \ldots & \varphi_{1}\left(s_{N}\right) \\
\varphi_{2}\left(s_{1}\right) & \varphi_{2}\left(s_{2}\right) & \ldots & \varphi_{2}\left(s_{N}\right) \\
\ldots & \ldots & \ldots & \ldots \\
\varphi_{N}\left(s_{1}\right) & \ldots & \ldots & \varphi_{N}\left(s_{N}\right)
\end{array}\right|
$$

where $\boldsymbol{s}_{i}=\boldsymbol{r}_{i}-\boldsymbol{r}_{\mathrm{p}}$.

Owing to the smallness of $v_{\boldsymbol{k}}(\boldsymbol{s})$ forbidding scattering to the states inside the Fermi sphere is superfluous since this introduces no change in $\Psi$.

Equation (1) for $\psi_{\boldsymbol{k}}(\boldsymbol{s})$ was solved in Born approximation assuming as zero approximation for $\psi_{\boldsymbol{k}}(\boldsymbol{s})$ - the function $\frac{w(s)}{\sqrt{\Omega}} \exp (\mathrm{i} \boldsymbol{k s})$. The potential $V(s)$ was chosen in the form as:

$$
V(s)=V_{0}(s)+v(s),
$$

where $V_{0}(s)$ is the self-consistent potential obtained from Eqs. $(12,13)$ and $v(s)$ is a small correction computed self-consistently. This approach allowed us to obtain the annihilation rate as a function of $r_{s}$, the momentum distribution of annihilation quanta and the correlation energy [3-5].

\section{The modified PHNC approach}

The function $w(s)$ obtained from Eqs. $(12,13)$ is a quite good choice to be used in the form (16) of the wave function of the system. However there is no reason to suppose that this is the best one. In order to optimize the function $w(s)$ it is necessary to find some criteria which could help to appreciate how good a choice is. The annihilation rates of Boroński and Nieminen [15] are somewhat lower than the ones following from PHNC. They are the ones to show the best agreement with experiment. They are based on the calculations of Lantto [9] which use the very complicated Fermi HNC method of solution and start from a not very convincing trial function. Monte-Carlo calculations using this trial function led to markedly different results for the annihilation rates moreover exhibiting unphysical features. So the formula of Boroński and Nieminen [15] should rather be treated as an empirical one. It is a challenging project to obtain similar results using the methods of many-body theory but free of the deficiencies of the Lantto calculations.

\subsection{The density of the screening cloud}

As shown in Ref. [3] in the case of weak nonorthogonality of the wave functions $\psi_{\boldsymbol{k}}$, we can write the electron density in the form as:

$$
\rho(s)=w^{2}(s) \rho_{0}+\delta \rho^{3}(s)-\delta \rho^{1}(s)-\delta \rho^{2}(s)=w^{2}(s) \rho_{0}+\delta \rho(s),
$$


where

$$
\begin{aligned}
& \delta \rho^{1}(s)=2 \sum_{\substack{\boldsymbol{k} \neq \boldsymbol{k}^{\prime} \\
\text { occ }}} A_{\boldsymbol{k}^{\prime} \boldsymbol{k}}^{1} \psi_{\boldsymbol{k}^{\prime}}^{*}(s) \psi_{\boldsymbol{k}}(\boldsymbol{s}), \\
& \delta \rho^{2}(s)=2 \sum_{\substack{\boldsymbol{k} \neq \boldsymbol{k}^{\prime} \\
\text { occ }}} A_{\boldsymbol{k}^{\prime} \boldsymbol{k}}^{2} \psi_{\boldsymbol{k}^{\prime}}^{*}(\boldsymbol{s}) \psi_{\boldsymbol{k}}(\boldsymbol{s}), \\
& \delta \rho^{3}(s)=\frac{2 w^{2}(s)}{\Omega} \sum_{\underset{\boldsymbol{k}}{\mathrm{occ}}}\left[v_{\boldsymbol{k}}(\boldsymbol{s}) \exp (-\mathrm{i} \boldsymbol{k} \boldsymbol{s})+v_{\boldsymbol{k}}^{*}(\boldsymbol{s}) \exp (\mathrm{i} \boldsymbol{k} \boldsymbol{s})\right] \\
& A_{\boldsymbol{k}^{\prime} \boldsymbol{k}}=A_{\boldsymbol{k}^{\prime} \boldsymbol{k}}^{1}+A_{\boldsymbol{k}^{\prime} \boldsymbol{k}}^{2}, \\
& A_{\boldsymbol{k}^{\prime} \boldsymbol{k}}^{1}=\frac{1}{\Omega} \int \mathrm{d} \boldsymbol{s}^{\prime}\left[w^{2}\left(s^{\prime}\right)-1\right] \exp \left(\mathrm{i}\left(\boldsymbol{k}^{\prime}-\boldsymbol{k}\right) \boldsymbol{s}^{\prime}\right), \\
& A_{\boldsymbol{k}^{\prime} \boldsymbol{k}}^{2}=\frac{1}{\Omega} \int \mathrm{d} \boldsymbol{s}^{\prime}\left[v_{\boldsymbol{k}^{\prime}}\left(\boldsymbol{s}^{\prime}\right) \exp \left(-\mathrm{i} \boldsymbol{k} \boldsymbol{s}^{\prime}\right)+v_{\boldsymbol{k}}^{*}\left(\boldsymbol{s}^{\prime}\right) \exp \left(\mathrm{i} \boldsymbol{k}^{\prime} \boldsymbol{s}^{\prime}\right)\right]
\end{aligned}
$$

We have [1]

$$
\delta \rho^{1}(s)=\frac{w^{2}(s) k_{\mathrm{F}}^{4}}{4 \pi^{3} s} \int_{0}^{\infty} \mathrm{d} s^{\prime} s^{\prime}\left[w^{2}\left(s^{\prime}\right)-1\right]\left[f\left(k_{\mathrm{F}}\left(s-s^{\prime}\right)\right)-f\left(k_{\mathrm{F}}\left(s+s^{\prime}\right)\right)\right],
$$

where

$$
f(x)=\frac{1}{x^{2}}\left(1-\frac{\sin (2 x)}{x}+\frac{1-\cos (2 x)}{2 x^{2}}\right) .
$$

The remaining terms in the electron density depend on the solution of Eq. (1). Solving it in Born approximation [3] we have in Eq. (11)

$$
\begin{aligned}
& A_{\boldsymbol{k}}(\boldsymbol{q})=\frac{\Phi_{1}(q) k \cos \theta+\Phi_{2}(q)}{(\boldsymbol{k}+\boldsymbol{q})^{2} / 2+q^{2} / 2-k^{2} / 2}, \\
& \Phi_{1}(q)=\frac{4 \pi}{q^{2}} \int_{0}^{\infty} \mathrm{d} s W(s)(\sin (q s)-q s \cos (q s)), \\
& \Phi_{2}(q)=-\frac{4 \pi}{q} \int_{0}^{\infty} \mathrm{d} s s v(s) \sin (q s) .
\end{aligned}
$$

$\theta$ is the angle between $\boldsymbol{k}$ and $\boldsymbol{q}$,

$$
W(s)=\frac{1}{w} \frac{\mathrm{d} w}{\mathrm{~d} s},
$$

$v(s)=V(s)-V_{0}(s)$ and is unknown, $\Omega$ is the volume of the sample.

Now we can write

$$
\delta \rho^{3}(s)-\delta \rho^{2}(s)=\frac{w^{2}(s)}{2 \pi^{4} s} \int_{0}^{\infty} \mathrm{d} q\left[\Phi_{1}(q) K_{1}(q)+\Phi_{2}(q) K_{2}(q)\right] \sin (q s),
$$

where 


$$
\begin{aligned}
& K_{1}(q)=\left\{\begin{array}{lll}
\frac{k_{\mathrm{F}}^{2}}{2} \phi\left(\frac{q}{k_{\mathrm{F}}}\right) & \text { for } & \frac{q}{k_{\mathrm{F}}}>2, \\
\frac{k_{\mathrm{F}}^{2}}{2} \zeta\left(\frac{q}{k_{\mathrm{F}}}\right) & \text { for } & \frac{q}{k_{\mathrm{F}}}<2,
\end{array}\right.
\end{aligned}
$$

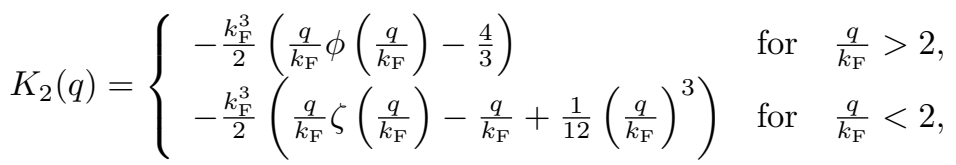

$$
\begin{aligned}
& \phi(x)=\left(1-x^{2}\right) \ln \left(\frac{1+1 / x}{1-1 / x}\right)+2 x, \\
& \zeta(x)=\ln (1+x)-x^{2} \ln (2+2 / x)+x+\frac{x^{2}}{2} .
\end{aligned}
$$

$k_{\mathrm{F}}$ is the Fermi wave vector.

Let us note that the total electron density around the positron is expressed partly in terms of the unknown function $\Phi_{2}(q)$ defined by Eq. (29). This function in real space corresponds to the unknown correction $v(s)$ to the electron-positron potential.

\subsection{Modification of the function $w(s)$}

Our aim is to express $w(s)$ in a suitable form which at the same time would be sufficiently flexible. It would be particularly desirable if $w(s)$ as the density amplitude led to a total screening charge neutralizing the charge of the positron. Such a requirement is satisfied by the solution of Eq. (12) with the potential

$$
V_{0}(\boldsymbol{s})=-\frac{1}{s}+\int \mathrm{d} \boldsymbol{s}^{\prime} \frac{w^{2}\left(s^{\prime}\right)-\rho_{0}}{\left|\boldsymbol{s}-\boldsymbol{s}^{\prime}\right|}+p(s)\left(V_{\mathrm{xc}}\left\{w^{2}(s) \rho_{0}\right\}-V_{\mathrm{xc}}\left\{\rho_{0}\right\}\right) .
$$

We estimate that a sufficient flexibility of the approach will be provided by $p(s)$ in the following form:

$$
p(s)=A \exp (-B s)+C .
$$

The form (36) of the potential $V_{0}(s)$ does not mean any assumption concerning the exchange-correlation potential. This is just a way of obtaining a parameter dependent function $w(s)$.

\subsection{Computation of the potential correction}

We will proceed in a way similar to that adopted in Ref. [3]. Let us define $\Delta V_{\mathrm{xc}}\{\rho(s)\}$ as

$$
\Delta V_{\mathrm{xc}}\{\rho(s)\}=V_{\mathrm{xc}}\{\rho(s)\}-V_{\mathrm{xc}}\left\{\rho_{0}\right\} .
$$

The potentials $V(s)$ and $V_{0}(s)$ satisfy the Poisson equations

$$
\begin{aligned}
& \nabla^{2}\left(V(s)-\Delta V_{\mathrm{xc}}(\rho(s))\right)=4 \pi\left[\delta(s)-\left(\rho(s)-\rho_{0}\right)\right] \\
& \nabla^{2}\left(V_{0}(s)-p(s) \Delta V_{\mathrm{xc}}\left\{w^{2}(s) \rho_{0}\right\}\right)=4 \pi\left[\delta(s)-\left(w^{2}(s)-1\right) \rho_{0}\right] .
\end{aligned}
$$

Subtracting (40) from (39) we get 


$$
\nabla^{2} v(s)-\nabla^{2}\left(\Delta V_{\mathrm{xc}}\{\rho(s)\}-p(s) \Delta V_{\mathrm{xc}}\left\{w^{2}(s) \rho_{0}\right\}\right)=-4 \pi \delta \rho(s) .
$$

We have from Eq. (38)

$$
\begin{gathered}
\Delta V_{\mathrm{xc}}\{\rho(s)\}=\Delta V_{\mathrm{xc}}\left\{w^{2}(s) \rho_{0}\right\}+\left.\frac{\mathrm{d}}{\mathrm{d} \rho}\left\{\Delta V_{x c}\right\}\right|_{\rho=w^{2}(s) \rho_{0}} \delta \rho(s) \\
=\Delta V_{\mathrm{xc}}\left\{w^{2}(s) \rho_{0}\right\}+\left.\frac{\mathrm{d} V_{\mathrm{xc}}}{\mathrm{d} \rho}\right|_{\rho=w^{2}(s) \rho_{0}} \delta \rho(s) .
\end{gathered}
$$

This leads to the equation

$$
\nabla^{2} v(s)-\nabla^{2}\left\{[1-p(s)] \Delta V_{\mathrm{xc}}\left\{w^{2}(s) \rho_{0}\right\}\right\}-\nabla^{2} v_{\mathrm{xc}}(s)=-4 \pi \delta \rho(s)
$$

where

$$
v_{\mathrm{xc}}(s)=\left.\frac{\mathrm{d} V_{\mathrm{xc}}}{\mathrm{d} \rho}\right|_{\rho=w^{2}(s) \rho_{0}} \delta \rho(s)=f_{\mathrm{xc}}(s) \delta \rho(s) .
$$

After multiplying this equation by $\pi^{3} s$ we get

$$
\begin{aligned}
& \pi^{3} s \nabla^{2} v(s)-\pi^{3} s \nabla^{2}\left\{[1-p(s)] \Delta V_{\mathrm{xc}}\left\{w^{2}(s) \rho_{0}\right\}\right\}-\pi^{3} s \nabla^{2}\left[f_{\mathrm{xc}}(s) \delta \rho(s)\right] \\
& \quad=-4 \pi^{4} s \delta \rho(s) .
\end{aligned}
$$

According to [3] $\delta \rho(s)$ can be expressed in the form as:

$$
\delta \rho(s)=-\frac{1}{4 \pi^{4} s}\left(L(s)+2 \pi k_{\mathrm{F}}^{2} w^{2}(s) \int_{0}^{\infty} \mathrm{d} s^{\prime} \mathcal{K}\left(s, s^{\prime}\right) s^{\prime} v\left(s^{\prime}\right)\right),
$$

where

$$
\begin{aligned}
L(s) & =2 w^{2}(s)\left\{\frac{\pi k_{\mathrm{F}}^{4}}{2} \int_{0}^{\infty} \mathrm{d} s^{\prime} s^{\prime}\left[w^{2}\left(s^{\prime}\right)-1\right]\left[f\left(k_{\mathrm{F}}\left(s-s^{\prime}\right)\right)-f\left(k_{\mathrm{F}}\left(s+s^{\prime}\right)\right)\right]\right. \\
& \left.-\int_{0}^{\infty} \mathrm{d} q \Phi_{1}(q) K_{1}(q) \sin (q s)\right\} .
\end{aligned}
$$

$\mathcal{K}\left(s, s^{\prime}\right)$ is defined as

$$
\mathcal{K}\left(s, s^{\prime}\right)=M\left(\left|s-s^{\prime}\right| k_{\mathrm{F}}\right)-M\left(\left(s+s^{\prime}\right) k_{\mathrm{F}}\right),
$$

where

$$
\begin{aligned}
& M(a)=M_{1}(a)+M_{2}(a) \\
& M_{1}(a)=\int_{0}^{2} \mathrm{~d} x m_{1}(x) \cos (a x), \\
& M_{2}(a)=\int_{2}^{\infty} \mathrm{d} x m_{2}(x) \cos (a x), \\
& m_{1}(x)=\frac{1}{x} \ln (1+x)-x \ln (2+2 / x)+1+\frac{x}{2}, \\
& m_{2}(x)=\left(\frac{1}{x}-x\right) \ln \left(\frac{1+1 / x}{1-1 / x}\right)+2 .
\end{aligned}
$$


Defining the function $Z(s)$ as $Z(s)=s v(s)$ we get from Eqs. $(45,46)$ the integro-differential equation

$$
\begin{aligned}
& \pi^{3} Z^{\prime \prime}(s)-2 \pi k_{\mathrm{F}}^{2} w^{2}(s) \int_{0}^{\infty} \mathrm{d} s^{\prime} \mathcal{K}\left(s, s^{\prime}\right) Z\left(s^{\prime}\right) \\
& \quad+\frac{k_{\mathrm{F}}^{2}}{2} \frac{\mathrm{d}^{2}}{\mathrm{~d} s^{2}}\left[f_{\mathrm{xc}}(s) w^{2}(s) \int_{0}^{\infty} \mathrm{d} s^{\prime} \mathcal{K}\left(s, s^{\prime}\right) Z\left(s^{\prime}\right)\right]=\Sigma(s),
\end{aligned}
$$

where

$$
\Sigma(s)=L(s)-\frac{1}{4 \pi} \frac{\mathrm{d}^{2}}{\mathrm{~d} s^{2}}\left[f_{\mathrm{xc}}(s) L(s)\right]+\pi^{3} \frac{\mathrm{d}^{2}}{\mathrm{~d} s^{2}}\left\{[1-p(s)] s \Delta V_{\mathrm{xc}}\left\{w^{2}(s) \rho_{0}\right\}\right\} .
$$

\section{Criterion for the optimal function $w(s)$}

The density $\rho(s)$ depends on the functions $w(s)$ and $v_{\boldsymbol{k}}(\boldsymbol{s})$ and is expressed by the formula (18). However the functions $v_{\boldsymbol{k}}(\boldsymbol{s})$ depend on whether we compute them according to PHNC [formulae (11) and (27-30)] or using the potential $V(s)$ in Eq. (1). In the last case, using Eqs. $(9,14,15)$ we have

$$
v_{\boldsymbol{k}}(\boldsymbol{s})=\exp \left(\mathrm{i} \frac{\boldsymbol{k}}{2} \boldsymbol{s}\right) \sum_{l=0}^{l_{N}}(2 l+1) i^{l} \gamma_{l}\left(\frac{k}{2}, s\right) P_{l}(\cos \vartheta),
$$

where we get for $l \leq l_{M}$

$$
\gamma_{l}\left(\frac{k}{2}, s\right)=\exp \left(\mathrm{i} \delta_{l}\left(\frac{k}{2}\right)\right) \frac{b_{l}\left(\frac{k}{2}, s\right)}{w(s)}-j_{l}\left(\frac{k}{2} s\right)
$$

and for $l>l_{M}$

$$
\gamma_{l}\left(\frac{k}{2}, s\right)=\left(\frac{1}{w(s)}-1\right) j_{l}\left(\frac{k}{2} s\right) .
$$

While $l$ increases the function $j_{l}\left(\frac{k}{2} s\right)$ starts to become big for higher and higher values of $s$ where the coefficient $\frac{1}{w(s)}-1$ in Eq. (58) is small. This limits the value of $l_{N}$.

In the expression (18) for $\rho(s)$ only the part $\delta \rho^{3}(s)-\delta \rho^{2}(s)$ depends on $v_{\boldsymbol{k}}(s)$. So it could be different depending on whether we compute it using the formula (11) for $v_{\boldsymbol{k}}(\boldsymbol{s})$. We obtain in this way the function $f(s)$. Or whether we use for this purpose the formula (56). We obtain in this case the function $g(s)$. If $f(s)$ and $g(s)$ differ strongly from each other this means that the assumptions underlying the PHNC approach are not quite satisfied. A function $w(s)$ leading to similar functions $f(s)$ and $g(s)$ is more satisfactory than the one providing very different functions.

\subsection{Computation of $g(s)$}

The function $f(s)$ is given by the formulae (46) and (25). So here we will show how to compute the function $g(s)$. 
We have

$$
g(s)=\delta \rho_{\mathrm{KS}}^{3}(s)-\delta \rho_{\mathrm{KS}}^{2}(s),
$$

where the subscript KS (Kohn-Sham) indicates that in the formulae (21) and (24) the form (56) of $v_{\boldsymbol{k}}(\boldsymbol{s})$ is used.

The definition of $\delta \rho^{3}(s)$ is given by Eq. (21).

Introducing $v_{\boldsymbol{k}}(\boldsymbol{s})$ in the form (56) into the formula (21) we get

$$
\delta \rho_{\mathrm{KS}}^{3}(s)=\frac{2 w^{2}(s)}{\pi^{2}} \sum_{l=0}^{l_{N}}(2 l+1) \int_{0}^{k_{\mathrm{F}}} k^{2} \mathrm{~d} k \operatorname{Re}\left[\gamma_{l}\left(\frac{k}{2}, s\right)\right] j_{l}\left(\frac{k}{2} s\right) .
$$

In computing $\delta \rho_{\mathrm{KS}}^{2}(s)$ we use the formula (20) in the form as:

$$
\delta \rho^{2}(s)=\frac{2 w^{2}(s)}{\Omega} \sum_{\substack{\boldsymbol{k} \neq \boldsymbol{k}^{\prime} \\ \text { oc }}} A_{\boldsymbol{k}^{\prime} \boldsymbol{k}}^{2} \exp \left(\mathrm{i}\left(\boldsymbol{k}-\boldsymbol{k}^{\prime}\right) \boldsymbol{s}\right) .
$$

Using the formula (56) for $v_{\boldsymbol{k}}(\boldsymbol{s})$ while computing $A_{\boldsymbol{k}^{\prime} \boldsymbol{k}}^{2}$ we get

$$
\begin{aligned}
& \delta \rho_{\mathrm{KS}}^{2}(s)=\frac{2 w^{2}(s)}{\pi^{3}} \sum_{l=0}^{l_{N}}(2 l+1) \\
& \quad \times \int_{0}^{k_{\mathrm{F}}} \mathrm{d} k^{\prime} \int_{0}^{\infty} \mathrm{d} s^{\prime} \operatorname{Re}\left[\gamma_{l}\left(\frac{k^{\prime}}{2}, s^{\prime}\right)\right] K_{l}\left(k^{\prime}, s^{\prime}, s\right),
\end{aligned}
$$

where

$$
\begin{aligned}
& K_{l}\left(k^{\prime}, s^{\prime}, s\right)=k_{\mathrm{F}}^{3} k^{\prime 2} s^{\prime 2} \int_{-1}^{1} \mathrm{~d} t \frac{\sin \left(k_{\mathrm{F}} s_{1}\right)-k_{\mathrm{F}} s_{1} \cos \left(k_{\mathrm{F}} s_{1}\right)}{\left(k_{\mathrm{F}} s_{1}\right)^{3}} j_{l}\left(k^{\prime} s_{2}\right) P_{l}(\tilde{t}), \\
& s_{1}=\left(s^{2}+s^{\prime 2}-2 s s^{\prime} t\right)^{1 / 2}, \quad s_{2}=\left(s^{2}+\frac{s^{\prime 2}}{4}-s s^{\prime} t\right)^{1 / 2}
\end{aligned}
$$

and

$$
\tilde{t}=\frac{1}{s_{2}}\left(s t-\frac{s^{\prime}}{2}\right) .
$$

Let us remark that the function $K_{l}\left(k^{\prime}, s^{\prime}, s\right)$ is universal, i.e. it is independent of the physical ingredient $\gamma_{l}\left(\frac{k}{2}, s\right)$. This is of great help in numerical computations.

\subsection{Comparison of $f(s)$ and $g(s)$}

The potential obtained from solving Eq. (54) is a Kohn-Sham-like potential self-consistent in Born approximation. Our calculations will show to be consistent if solving exactly the Kohn-Sham-like equations (1) with this potential will lead approximately to the same electron distribution around the positron. In other words $f(s)$ and $g(s)$ should be close to each other. Since we cannot expect that these two functions will be identical, we must assume some numerical quantity which would express the similarity of the two functions by means of a single number. We believe that it is crucial that both functions generate the same contribution to the potential. Assuming that the self-consistent Born approximation leads to an electron density distribution around the positron equal $\rho_{1}(s)$ and a potential 
$V_{1}(s)$ while the exact solution of the Kohn-Sham equations with the same potential gives an electron distribution $\rho_{2}(s)$ which generates a potential $V_{2}(s)$ we find it logical to minimize with regard to $p(s)$ the integral

$$
\Delta\{p(s)\}=\int s^{2} \mathrm{~d} s\left\{V_{2}(s) \rho_{2}(s)-V_{1}(s) \rho_{1}(s)\right\}^{2} .
$$

We have

$$
\rho_{2}(s)=\rho_{1}(s)+g(s)-f(s)
$$

and (cf. [3])

$$
V_{i}(s)=-\frac{1}{s}+\int \mathrm{d} \boldsymbol{s}^{\prime} \frac{\rho_{i}\left(s^{\prime}\right)-\rho_{0}}{\left|s-s^{\prime}\right|}+V_{\mathrm{xc}}\left\{\rho_{i}, s\right\}-V_{\mathrm{xc}}\left\{\rho_{0}\right\} .
$$

This gives

$$
V_{2}(s)=V_{1}(s)+\int \mathrm{d} \boldsymbol{s}^{\prime} \frac{g\left(s^{\prime}\right)-f\left(s^{\prime}\right)}{\left|s-s^{\prime}\right|}+V_{\mathrm{xc}}\left\{\rho_{2}, s\right\}-V_{\mathrm{xc}}\left\{\rho_{1}, s\right\}
$$

and

$$
\Delta\{p(s)\}=\int s^{2} \mathrm{~d} s\left\{\left[V_{2}(s)-V_{1}(s)\right] \rho_{2}(s)+V_{1}(s)[g(s)-f(s)]\right\}^{2} .
$$

\section{Computations and results}

Computations consist in finding values of $A, B$, and $C$ in the formula (37) for $p(s)$ which minimize the integral (66). In general this leads to slightly lower annihilation rates than when determining $w(s)$ like in Ref. [3]. Of course, every set of values $A, B, C$ is suitable for PHNC calculations. The way to proceed is to try one by one different sets of values in order to find such sets for which $\Delta\{p(s)\}$ is particularly small.

We do not yet dispose of definitive results. Let us consider the case when $r_{s}=1.5$ and the radius of perturbation of the electron gas by the positron is limited to $R_{\max }=19.85$. Labelling 1 the case when $A=0, B=0$ and $C=1$, and 2 - the case when $A=-1.52, B=1.3$, and $C=4$, we have

$$
\frac{\Delta\left\{p_{1}(s)\right\}}{\Delta\left\{p_{2}(s)\right\}}=67.67
$$

Among the several dozens of sets we tried, the set of the case 2 yields a particularly low value of $\Delta\{p(s)\}$.

The corresponding density distributions $\rho(s)$ are shown in figures. The full curves show the PHNC (or modified PHNC) distributions. The dashed curves are obtained from solving the Kohn-Sham-like equations with the appropriate PHNC potential. In the case 1 (Fig. 1) the Kohn-Sham-like density of the screening cloud exceeds (unphysically) the PHNC distribution. In the case 2 the two distributions are to a large degree identical (Fig. 2). In Fig. 3 we compare the Kohn-Sham electron density of the case 2 to the PHNC result (case 1). We see that applying the function $p(s)$ of the case 2 leads on one side to a convincing screening cloud distribution (equality of the modified PHNC distribution and the Kohn-Sham 


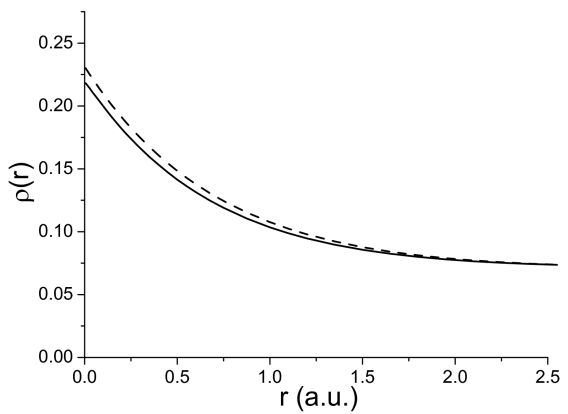

Fig. 1. The electron density distribution close to the positron - case 1: the PHNC result (full curve) and the Kohn-Sham distribution (dashed curve).

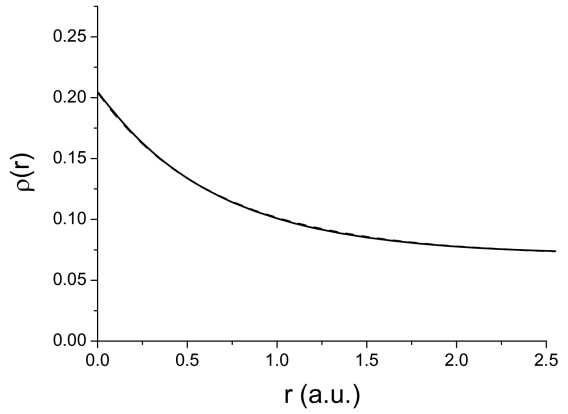

Fig. 2. The electron density distribution close to the positron - case 2: the PHNC result (full curve) and the Kohn-Sham distribution (dashed curve). The dashed curve is not visible because the two curves overlap.

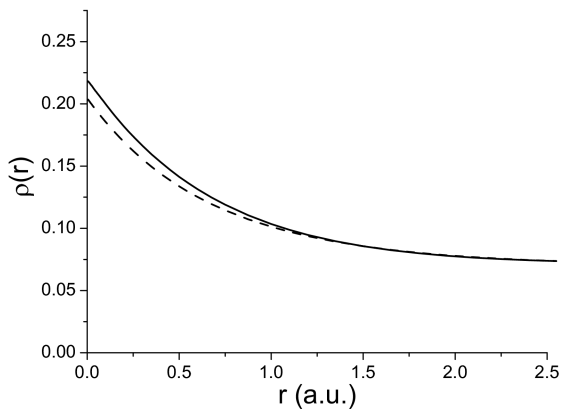

Fig. 3. The electron density distribution close to the positron: the PHNC result obtained in the case 1 (full curve) compared to the Kohn-Sham distribution obtained in the case 2 (dashed curve).

electron distribution following from the corresponding PHNC potential) and on the other side provides a lower annihilation rate. In the case 2 the Kohn-Sham annihilation rates are lower by $6.76 \%$ than the usual PHNC results. 


\section{Conclusions}

Computations are still under way. However some conclusions can be formulated at the present stage:

1. The results of PHNC calculations are remarkably stable with regard to modifications of the function $w(s)$, more precisely, with regard to changing the values of $A, B$, and $C$ in the formula (37) in order to minimize $\Delta\{p(s)\}$ in the formula (66).

2. The annihilation rates obtained by minimizing $\Delta\{p(s)\}$ are lower by a few percent than the ones following from using the potential (13) in the equation for $w(s)$, approaching in this way experimental expectations. More precise statements cannot be formulated yet.

\section{References}

[1] H. Stachowiak, E. Boroński, Phys. Rev. B 71, 245107 (2005).

[2] E. Boroński, H. Stachowiak, Physica B 366, 168 (2005).

[3] H. Stachowiak, Phys. Rev. B, 41, 12522 (1990).

[4] H. Stachowiak, J. Lach, Phys. Rev. B 48, 9828 (1993).

[5] E. Boroński, H. Stachowiak, Phys. Rev. B 57, 6215 (1998).

[6] H. Stachowiak, E. Boroński, Acta Phys. Pol. A 107, 541 (2005).

[7] L.I. Schiff, Quantum Mechanics McGraw-Hill, New York 1955, p. 125.

[8] S. Kahana, Phys. Rev. 129, 1622 (1963).

[9] L. Lantto, Phys. Rev. B 36, 5160 (1987).

[10] A. Kallio, P. Pietiläinen, L. Lantto, Phys. Scr. 25, 943 (1982); L. Lantto, private communication.

[11] P. Pietiläinen, A. Kallio, Phys. Rev. B 27, 224 (1983).

[12] J. Gondzik, H. Stachowiak, J. Phys. C 18, 5399 (1985).

[13] J.G. Zabolitzky, Phys. Rev. B 22, 2353 (1980).

[14] L. Hedin, B.J. Lundqvist, J. Phys. C 4, 2064 (1971).

[15] E. Boroński, R.M. Nieminen, Phys. Rev. B 34, 3820 (1986). 